\title{
EFECTOS GENÉTICOS ADITIVOS Y NO ADITIVOS EN CRUCES ROTACIONALES HOLSTEIN×JERSEY Y HOLSTEIN×PARDO SUIZO ${ }^{1}$
}

\author{
Bernardo Vargas-Leitón ${ }^{2}$, Juan José Romero-Zúñiga ${ }^{2}$
}

\begin{abstract}
RESUMEN
Efectos genéticos aditivos y no aditivos en cruces rotacionales Holstein $\times$ Jersey y Holstein $\times$ Pardo Suizo. El objetivo del presente estudio fue cuantificar el impacto de efectos genéticos aditivos y no aditivos sobre días abiertos (DA), edad a primer parto (EPP), vida productiva (VP) y producción de leche por día $(\mathrm{KG})$ en cruces rotacionales Holstein $\times$ Jersey (HxJ) y Holstein $\times$ Pardo Suizo (HxPS). Los datos utilizados se colectaron en fincas lecheras de Costa Rica en el periodo entre 1990 a 2009. Los efectos fueron cuantificados utilizando dos métodos: un modelo estadístico de clasificación racial y un modelo genético de regresión sobre heterocigosidad esperada. Se observaron correlaciones mayores a 0,90 entre los rendimientos predichos por ambos métodos. Los porcentajes de heterosis directa para $\mathrm{H} \times \mathrm{J} v s$. $\mathrm{H} \times \mathrm{PS}$ fueron, respectivamente: $-6,4$ vs. $-2,2$ para DA; $-2,5$ vs. $-2,2$ para EPP, 4,4 vs. 12,2 para VP, y 3,2 vs. 3,8 para KG. Según el modelo genético los efectos aditivos directos fueron significativos $(\mathrm{P}<0,05)$ para todos los rasgos excepto VP en $\mathrm{H} \times \mathrm{PS}$; mientras que los efectos aditivos maternos fueron significativos $(\mathrm{P}<0,05)$ para $\mathrm{VP}$ y $\mathrm{KG}$ en ambos cruces rotacionales. La heterosis directa fue significativa $(\mathrm{P}<0,05)$ para $\mathrm{DA}, \mathrm{EPP}$ y $\mathrm{KG}$ en $\mathrm{H} \times \mathrm{J}$; así como $\mathrm{EPP}, \mathrm{VP}$ y $\mathrm{KG}$ en $\mathrm{H} \times \mathrm{PS}$. La heterosis materna fue significativa $(\mathrm{P}<0,05)$ para EPP y $\mathrm{KG}$ en $\mathrm{H} \times \mathrm{J}$ y para $\mathrm{KG}$ en $\mathrm{H} \times \mathrm{PS}$. El efecto de recombinación fue significativo $(\mathrm{P}<0,05)$ para EPP y $\mathrm{KG}$ en $\mathrm{H} \times \mathrm{J}$; así como para $\mathrm{KG}$ en $\mathrm{H} \times \mathrm{PS}$. En general, tanto los efectos genéticos aditivos como no aditivos juegan un papel importante en estos dos cruces.
\end{abstract}

Palabras claves: Cruces lecheros, heterosis, rasgos funcionales, rasgos productivos.

\begin{abstract}
Additive and non additive genetic effects in Holstein $\times$ Jersey and Holstein $\times$ Brown Swiss rotational crosses. The objective of this study was to assess the impact of additive and non-additive genetic effects on open days (DO), age at first calving (AFC), herd-life (HL) and daily milk yield (DMY) in Holstein $\times$ Jersey $(\mathrm{H} \times \mathrm{J})$ and Holstein $\times$ Brown Swiss $(\mathrm{H} \times \mathrm{BS})$ rotational crosses. Data were collected in dairy farms from Costa Rica from 1990 to 2009. Effects were assessed by using two models: a statistical model with breed-type as fixed effect and a genetic model based on regression on expected heterocigocity. Correlations higher than 0.90 were obtained between performance predicted by the genetic model and the least squares means obtained by the statistical model. Direct heterosis percentages for $\mathrm{H} \times \mathrm{J}$ vs. $\mathrm{H} \times \mathrm{BS}$ crosses were, respectively: -6.4 vs. -2.2 for $\mathrm{DO}$; -2.5 vs. -2.2 for AFC, 4.4 vs. 12.2 for HL, and 3.2 vs. 3.8 for DMY. According to the genetic model, direct additive effects were significant $(\mathrm{P}<0.05)$ for all traits except $\mathrm{HL}$ in $\mathrm{H} \times \mathrm{BS}$; whereas maternal additive effects were significant for HL and DMY in both crosses. Direct heterosis was significant $(\mathrm{P}<0.05)$ for $\mathrm{DO}, \mathrm{AFC}$ and $\mathrm{DMY}$ in $\mathrm{H} \times \mathrm{J}$, as for $\mathrm{AFC}$, $\mathrm{HL}$ and DMY in $\mathrm{H} \times \mathrm{BS}$. Maternal heterosis was significant $(\mathrm{P}<0.05)$ for $\mathrm{AFC}$ and $\mathrm{DMY}$ in $\mathrm{H} \times \mathrm{J}$; and for $\mathrm{DMY}$ in $\mathrm{H} \times \mathrm{BS}$. Recombination effect was significant $(\mathrm{P}<0.05)$ for $\mathrm{AFC}$ and $\mathrm{DMY}$ in $\mathrm{H} \times \mathrm{J}$; and for $\mathrm{DMY}$ in $\mathrm{H} \times \mathrm{BS}$. In general, both additive and non-additive genetic effects play an important role in these two crosses.
\end{abstract}

Key words: Dairy crosses, heterosis, functional traits, productive traits.

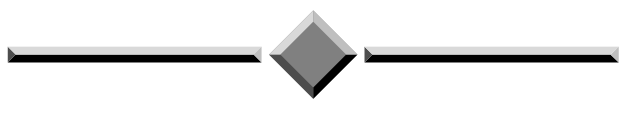

1 Recibido: 24 de mayo, 2010. Aceptado: 18 de noviembre, 2010. Este estudio es parte de los resultados de un proyecto de investigación ejecutado por los autores en la Universidad Nacional.

2 Posgrado Regional en Ciencias Veterinarias Tropicales, Universidad Nacional, Costa Rica. Apdo Postal 304-3000. bvargas@medvet.una. ac.cr; jromero@medvet.una.ac.cr 


\section{INTRODUCCIÓN}

Los cruces entre razas han sido utilizados por largo tiempo en la producción bovina, tanto con fines lecheros como cárnicos; son importantes ya que, además de aprovechar las diferencias genéticas aditivas entre razas, también permiten hacer uso de la heterosis y de la complementariedad (Swan y Kinghorn 1992, McAllister et al. 1994, McAllister 2002, Casell y McAllister 2007). La heterosis es atribuida a la acción conjunta de factores genéticos no aditivos, principalmente efectos de dominancia. Una posible desventaja de los cruces es la pérdida de combinaciones epistáticas favorables por recombinación, lo que puede ocurrir en generaciones posteriores a la $\mathrm{F} 1$ obtenidas por intercruzamiento (Madalena 1993).

Tradicionalmente, en países de zonas templadas la producción de leche ha dependido casi en forma exclusiva del uso de razas puras altamente especializadas tales como la Holstein o la Jersey (Van Raden 1992). Generalmente se aduce que bajo circunstancias de manejo intensivo los cruces no son capaces de superar el rendimiento de las razas puras. Sin embargo, en países de tradición lechera tales como Nueva Zelanda, más del $20 \%$ del hato lechero está constituido por cruces, principalmente Holstein $\times$ Jersey (Ahlborn-Breier y Hohenboken 1991, López-Villalobos et al. 2000a).

Los cruces entre razas lecheras están siendo cada vez más comunes debido a problemas asociados al uso intensivo de las razas puras, tales como el aumento en la consanguinidad y la reducción paulatina en los índices de rendimiento reproductivo y longevidad (Van Raden y Sanders 2003, Cassell y McAllister 2007, Madalena 2008). Otro factor que contribuye al uso de los cruces es la migración hacia sistemas de pago por componentes, los cuales penalizan más la producción de fluído (Cassell y McAllister 2007).

En Costa Rica es bastante común el uso de cruces entre razas lecheras, principalmente entre la raza mayoritaria Holstein y las Jersey o Pardo Suizo. De acuerdo con datos procedentes de más de 600 fincas lecheras, la proporción de animales cruzados dentro de la población de ganado lechero es mayor al $20 \%$. La mayoría de las fincas que practican cruzamientos utilizan un sistema de cruces rotacional, alternando el uso de toros puros de las dos razas utilizadas. No existe un estudio que evalúe la eficacia a nivel local del uso de estos cruces en comparación con las razas puras. El objetivo del presente estudio fue cuantificar el impacto de efectos genéticos aditivos y no aditivos en cruces rotacionales Holstein $\times$ Jersey y Holstein $\times$ Pardo Suizo.

\section{MATERIALES Y MÉTODOS}

La información utilizada para este estudio se obtuvo de la base de datos del Centro Regional de Informática para la Producción Animal Sostenible (CRIPAS) de la Universidad Nacional de Costa Rica (Noordhuizen y Buurman 1984, Pérez et al. 1989). Esta base de datos concentra información proveniente de hatos localizados en las principales regiones lecheras de Costa Rica y distribuidos entre las siguientes zonas de vida: Bosque Muy Húmedo Tropical (40,5\%), Bosque Muy Húmedo- Premontano (27,0\%), Bosque Muy Húmedo-Montano Bajo (17,5\%) y el restante $15 \%$ distribuido dentro de las distintas unidades naturales de la zona Bosque Húmedo (Tropical, Montano Bajo o Montano) (Holdridge 2000). Los hatos están en su mayoría adscritos a un programa de monitoreo ejecutado por una cooperativa local y la información se centraliza para su análisis en CRIPAS. La información analizada abarca un periodo de aproximadamente 20 años (1990-2009). Sin embargo, existieron amplias diferencias en cantidad de años de seguimiento entre los distintos hatos. Los hatos incluidos corresponden a sistemas de producción de lechería especializada con ordeño mecánico, alta proporción y uso generalizado de alimentos concentrados en la dieta, aunque no exclusivo e inseminación artificial.

En el presente estudio se excluyeron los hatos que tenían menos de $15 \%$ de animales cruzados, con el fin de asegurar una adecuada representatividad de los cruces dentro de las explotaciones y evitar además la inclusión de hatos puros. Además se requirió un mínimo de cinco animales por hato, resultando al final un total de 270 hatos. Dentro de los hatos participantes se realizó una selección inicial de las vacas que tenían ambos padres identificados con su respectiva composición racial. Posteriormente, se seleccionaron los grupos raciales que se presentan con mayor frecuencia en las fincas, debido a que muchas de las fincas practican un sistema de cruzamiento rotacional en transición, los cruces más frecuentes que se encontraron fueron entre toros puros (vía inseminación artificial) $\times$ hembras puras, F1 o 3/4 de raza (Cuadro 1). Se identificaron un 
total de 1542 toros Holstein, 1233 toros Jersey y 245 toros Pardo Suizo.

Los datos fueron analizados asumiendo dos diferentes modelos, un modelo estadístico de clasificación racial y un modelo genético basado en regresión sobre fracciones esperadas de heterocigosidad, según el procedimiento sugerido por Madalena (2001) para validación de modelos genéticos.

El modelo estadístico de clasificación racial fue el siguiente:

$$
Y=u+H A E+G R A Z+e
$$

donde:

$Y=$ Variables de respuesta. Se consideraron los rasgos: Edad a primer parto (meses), días abiertos (días), vida productiva (meses) definida como periodo transcurrido entre el primer parto y el descarte; y kilogramos de leche por día de lactancia,

$u=$ Media general,

$H A E=$ Efecto aleatorio formado por clases combinadas de hato, año y época de nacimiento de la vaca,

$G R A Z=$ Efecto fijo de grupo racial (Cuadro 1),

$e=$ Error residual aleatorio.
Para los rasgos días abiertos y kilogramos de leche, se incluyó el efecto:

$L A X E D=$ Efecto fijo formado por clases combinadas de número de lactancia (1 hasta $\geq 5)$ y edad en años $(2$ hasta $\geq 8)$ de la vaca.

Asimismo, para el rasgo $\mathrm{kg}$ de leche se incluyó también los efectos:

$P E R=$ Efecto lineal de la covariable periodo (en semanas) de la lactancia,

PER2 = Efecto cuadrático de la covariable periodo de la lactancia.

Para todos los rasgos analizados se eliminaron los valores extremos o biológicamente improbables utilizando como criterio 3,5 desviaciones estándares con respecto al promedio general observado. Este modelo se resolvió utilizando el procedimiento MIXED del programa estadístico SAS (SAS 2009).

El porcentaje de heterosis directa o individual se estimó a partir de las medias de mínimos cuadrados (MMC), obtenidas del modelo anterior para cada

Cuadro 1. Clasificación de los grupos raciales ${ }^{1}$ más frecuentes en la población bajo estudio (base de datos de fincas lecheras del Centro Regional de Informática para la Producción Animal Sostenible (CRIPAS), Universidad Nacional, Costa Rica, periodo 1990-2009.

\begin{tabular}{|c|c|c|c|}
\hline $\begin{array}{l}\text { Grupo } \\
\text { racial }\end{array}$ & $\begin{array}{c}\text { Raza } \\
\text { paterna }\end{array}$ & $\begin{array}{c}\text { Raza } \\
\text { materna }\end{array}$ & Caracterización de grupo racial \\
\hline $\mathrm{J} 8$ & $\mathrm{~J} 8$ & $\mathrm{~J} 8$ & Raza Jersey pura \\
\hline H2J6 & $\mathrm{J} 8$ & $\mathrm{H} 4 \mathrm{~J} 4$ & Retrocruce toro Jersey $\times$ vaca F1 Holstein $\times$ Jersey \\
\hline H3J5 & $\mathrm{J} 8$ & H6J 2 & Retrocruce recíproco toro Jersey $\times$ vaca 3/4Holstein $1 / 4$ Jersey \\
\hline H4J4 & H8 o J8 & J8 o H8 & F1 Holstein $\times$ Jersey (cruces recíprocos) \\
\hline $\mathrm{H} 5 \mathrm{~J} 3$ & $\mathrm{H} 8$ & H2J6 & Retrocruce recíproco toro Holstein×vaca $1 / 4$ Holstein $3 / 4$ Jersey \\
\hline $\mathrm{H} 6 \mathrm{~J} 2$ & $\mathrm{H} 8$ & $\mathrm{H} 4 \mathrm{~J} 4$ & Retrocruce toro Holstein×vaca F1 Holstein×Jersey \\
\hline $\mathrm{H} 8$ & $\mathrm{H} 8$ & $\mathrm{H} 8$ & Raza Holstein pura \\
\hline H6PS2 & $\mathrm{H} 8$ & H4PS4 & Retrocruce toro Holstein $\times$ vaca F1 Holstein $\times$ P. Suizo \\
\hline H5PS3 & H8 & H2PS6 & Retrocruce recíproco toro Holstein $\times$ vaca $1 / 4$ Holstein $3 / 4$ P. Suizo \\
\hline H4PS4 & H8 o PS8 & PS8 o H8 & F1 Holstein $\times$ Pardo Suizo (cruces recíprocos) \\
\hline H3PS5 & PS8 & H6PS2 & Retrocruce recíproco toro Pardo Suizoxvaca $3 / 4$ Holstein $1 / 4$ P.Suizo \\
\hline H2PS6 & PS8 & H4PS4 & Retrocruce toro Pardo Suizo×vaca F1 Holstein $\times$ P. Suizo \\
\hline PS8 & PS8 & PS8 & Raza Pardo Suizo pura \\
\hline
\end{tabular}

${ }^{1}$ En el sistema de información utilizado la composición racial de los animales se describe en octavos de raza. 
grupo racial y cada uno de los rasgos, utilizando la siguiente fórmula:

$$
\% H=\frac{M M C_{F 1}-\overline{M M C}_{P}}{\overline{M M C}_{P}} \times 100
$$

donde:

$M M C_{F 1}=$ Media de mínimos cuadrados para el grupo racial H4J4 o H4PS4.

$\overline{M M C_{P}}=$ Promedio de la medias de los mínimos cuadrados de los grupos raciales paternos (H8 vs. J8 o PS8).

Posteriormente, los rasgos fueron analizados mediante el siguiente modelo genético:

$\mathrm{Y}=u+H A E+\beta_{1} A D \_J+\beta_{2} A M \_J+\beta_{3} H D \_J+$ $\beta_{4} H M_{-} J+\beta_{5} R \_J+\beta_{6} \mathrm{AD} \_\mathrm{PS}+\beta_{7} \mathrm{AM} \_\mathrm{PS}+\beta_{8} \mathrm{HD}$ $\mathrm{PS}+\beta_{9}$ HM_PS $+\beta_{10} \mathrm{R} \_\mathrm{PS}+e$

donde:

$Y=$ Variables de respuesta, como en modelo [1],

$u=$ Media general,

$H A E=$ Efecto aleatorio de clase de hato, año y época de nacimiento,

$A D \_J=$ Efecto de regresión lineal $\left(\beta_{1}\right)$ sobre fracción aditiva directa Jersey (cruce $\mathrm{H} \times \mathrm{J}$ ),

$A M_{\_} J=$ Efecto de regresión lineal $\left(\beta_{2}\right)$ sobre fracción aditiva materna Jersey (cruce $\mathrm{H} \times \mathrm{J}$ ),

$H D \_J=$ Efecto de regresión lineal $\left(B_{3}\right)$ sobre fracción de heterosis directa (cruce $\mathrm{H} \times \mathrm{J}$ ),

$H M_{-} J=$ Efecto de regresión lineal $\left(\beta_{4}\right)$ sobre fracción de heterosis materna (cruce $\mathrm{H} \times \mathrm{J}$ ),

$R \_J=$ Efecto de regresión lineal $\left(\beta_{5}\right)$ sobre fracción de recombinación (cruce $\mathrm{H} \times \mathrm{J}$ ),

$A D \_P S=$ Efecto de regresión lineal $\left(\beta_{6}\right)$ sobre fracción aditiva directa P. Suizo (cruce $\mathrm{H} \times \mathrm{PS}$ ),

$A M \_P S=$ Efecto de regresión lineal $\left(\beta_{7}\right)$ sobre fracción aditiva materna P.Suizo (cruce $\mathrm{H} \times \mathrm{PS}$ ),

$H D \_P S=$ Efecto de regresión lineal $\left(\beta_{8}\right)$ sobre fracción de heterosis directa (cruce $\mathrm{H} \times \mathrm{PS}$ ),

$H M_{-} P S=$ Efecto de regresión lineal $\left(\beta_{q}\right)$ sobre fracción de heterosis materna (cruce $\mathrm{H} \times \mathrm{PS}$ ),

$R \_P S=$ Efecto de regresión lineal $\left(\beta_{10}\right)$ sobre fracción de recombinación (cruce $\mathrm{H} \times \mathrm{PS}$ ),

$e=$ Error residual aleatorio.

En el modelo [3] también se adicionaron los efectos de LAXED, PER y PER2 en los rasgos días abiertos y kilogramos de leche según se describió para el modelo [1].

En el modelo genético los efectos aditivos, la heterosis y la recombinación correspondientes a $\mathrm{H} \times \mathrm{J}$ y $\mathrm{H} \times \mathrm{PS}$ se especificaron por separado, con el fin de obtener estimados específicos para cada cruce. La fracción genética aditiva (A), directa o materna, se especificó como desviación de la raza Holstein, la cual se utilizó como base de comparación. La fracción esperada de heterocigosidad $(\mathrm{H})$, directa o materna, se calculó de acuerdo con la fórmula siguiente (Van Raden y Sanders 2003):

$$
H=\left(l-\sum_{i=1}^{n} p_{s_{i}} p_{d_{i}}\right)
$$

Donde $\mathrm{p}_{\mathrm{si}}$ y $\mathrm{p}_{\mathrm{di}}$ son las fracciones de genes de la raza $i$ provenientes de cada uno de los padres. La fracción esperada de recombinación $R$ se calculó según la siguiente fórmula (Van Raden y Sanders 2003):

$$
R=\left(1-\frac{\sum_{i=1}^{n}\left(p_{s_{i}}{ }^{2}+p_{d_{i}}{ }^{2}\right)}{2}\right)
$$

Los valores esperados para las fracciones aditivas, de heterosis y recombinación de cada grupo racial calculados por las fórmulas 4 y 5 se especifican en el Cuadro 2.

El modelo genético fue resuelto utilizando el PROC MIXED del programa SAS (SAS 2009). Se obtuvieron los coeficientes de regresión para cada uno de los efectos y se evaluó su significancia estadística $\left(H_{0}: \beta=0, H_{A}: \beta \neq 0\right)$. Adicionalmente, se obtuvieron los rendimientos predichos para cada variable de respuesta y cada grupo racial a partir de la suma de productos entre los valores esperados de las fracciones genéticas (Cuadro 2) y sus coeficientes respectivos, asumiendo distribución uniforme del efecto LAXED y valores promedio para las covariables PER y PER2. Se compararon los rendimientos predichos por el modelo genético [2] contra las medias de mínimos cuadrados del modelo [1] con el fin de evaluar el ajuste del modelo genético a los datos obtenidos en campo.

Con el fin de integrar las diferencias observadas tanto en rasgos productivos como funcionales, se 
Cuadro 2. Valores esperados para fracciones genéticas aditivas directas (AD_J, AD_PS) y maternas (AM_J; AM_PS), heterosis directa (HD_J, HD_PS) y materna (HM_J, HM_PS) y recombinación (R_J, R_PS) para razas puras (Holstein,H8; Jersey,J8, y Pardo Suizo, PS8), cruces F1 (H4J4 y H4PS4) y otros cruces (H2J6, H6J2, H2PS6, H6PS2, H3J5, H5J3, H3PS5, H5PS3) Costa Rica, periodo 1990-2009.

\begin{tabular}{ccccccccccc}
\hline $\begin{array}{c}\text { Grupo } \\
\text { racial }\end{array}$ & AD_J & AM_J & HD_J & HM_J & R_J & AD_PS & AM_PS & HD_PS & HM_PS & R_PS \\
\hline J8 & 1,000 & 1,000 & 0,000 & 0,000 & 0,000 & 0,000 & 0,000 & 0,000 & 0,000 & 0,000 \\
H2J6 & 0,750 & 0,500 & 0,500 & 1,000 & 0,250 & 0,000 & 0,000 & 0,000 & 0,000 & 0,000 \\
H3J5 & 0,625 & 0,250 & 0,750 & 0,500 & 0,188 & 0,000 & 0,000 & 0,000 & 0,000 & 0,000 \\
H4J4 & 0,500 & 1,000 & 1,000 & 0,000 & 0,000 & 0,000 & 0,000 & 0,000 & 0,000 & 0,000 \\
H5J3 & 0,375 & 0,750 & 0,750 & 0,500 & 0,188 & 0,000 & 0,000 & 0,000 & 0,000 & 0,000 \\
H6J2 & 0,250 & 0,500 & 0,500 & 1,000 & 0,250 & 0,000 & 0,000 & 0,000 & 0,000 & 0,000 \\
H8 & 0,000 & 0,000 & 0,000 & 0,000 & 0,000 & 0,000 & 0,000 & 0,000 & 0,000 & 0,000 \\
H6PS2 & 0,000 & 0,000 & 0,000 & 0,000 & 0,000 & 0,250 & 0,500 & 0,500 & 1,000 & 0,250 \\
H5PS3 & 0,000 & 0,000 & 0,000 & 0,000 & 0,000 & 0,375 & 0,750 & 0,750 & 0,500 & 0,188 \\
H4PS4 & 0,000 & 0,000 & 0,000 & 0,000 & 0,000 & 0,500 & 1,000 & 1,000 & 0,000 & 0,000 \\
H3PS5 & 0,000 & 0,000 & 0,000 & 0,000 & 0,000 & 0,625 & 0,250 & 0,750 & 0,500 & 0,188 \\
H2PS6 & 0,000 & 0,000 & 0,000 & 0,000 & 0,000 & 0,750 & 0,500 & 0,500 & 1,000 & 0,250 \\
PS8 & 0,000 & 0,000 & 0,000 & 0,000 & 0,000 & 1,000 & 1,000 & 0,000 & 0,000 & 0,000 \\
\hline
\end{tabular}

calculó un estimado de producción de leche por día de vida $(\mathrm{KGV})$. Este rasgo refleja hasta cierto punto la eficiencia biológica de los grupos raciales involucrados, y se calculó con base en las medias de mínimos cuadrados obtenidas a partir del modelo estadístico, utilizando la fórmula 6. En esta fórmula KGV disminuye conforme aumenta la edad a primer parto y el intervalo entre éstos; y aumenta conforme se incrementa la vida productiva y la producción diaria:

$$
K G V=\frac{(V P-D S) x K G}{(E P P+V P)}
$$

$\mathrm{KGV}=$ Producción de leche $(\mathrm{kg})$ por día de vida, $\mathrm{VP}=$ MMC Vida productiva (en días),

DS = Estimado de días secos (improductivos) durante vida en el hato,

$\mathrm{DS}=$ ((intervalo entre partos - longitud de lactancia) $\times$ número de lactancias),

$\mathrm{KG}=\mathrm{MMC}$ para producción de leche por día, $\mathrm{EPP}=\mathrm{MMC}$ para edad a primer parto (días).

\section{RESULTADOS Y DISCUSIÓN}

Los días abiertos oscilaron entre 124,9 (H2J6) y 164,8 (H3PS5), la edad a primer parto entre 26,7 (H5PS3) y 30,2 (H8) meses, la vida productiva entre 29,9 (H5J3) y 43,5 (H4PS4) meses y la producción de leche entre 15,1 (J) y 18,6 (H8) kg/día (Cuadro 3). La información disponible para los cruces (H3J5, H5J3, H5PS3 y H3PS5) siempre fue más limitada en todos los rasgos, lo que incidió en la precisión de los parámetros del modelo obtenidos posteriormente.

Los resultados del modelo estadístico [1] y del modelo genético [2] fueron altamente concordantes para todos los rasgos analizados. Las medias de mínimos cuadrados según el modelo de clasificación (promedios y sus respectivos intervalos de confianza) para cada uno de los rasgos y grupos raciales se muestran en la Figura 1; se sobreponen los valores predichos por el modelo genético (línea continua).

Existe coincidencia entre los resultados de ambos modelos, siendo la correlación entre estimados de 90,7\% para días abiertos, 97,7\% para edad al parto, 
Cuadro 3. Medias aritméticas (X), error estándar de la media (E.E) y número de registros disponibles (n) para los rasgos de días abiertos, edad a primer parto, vida productiva y kg de leche/día en las razas puras (Holstein,H8; Jersey,J8, y Pardo Suizo, PS8), cruces F1 (H4J4 y H4PS4) y otros cruces (H2J6, H6J2, H2PS6, H6PS2, H3J5, H5J3, H3PS5, H5PS3). Costa Rica, periodo 1990-2009.

\begin{tabular}{lcccccccccccc}
\hline Grupo & \multicolumn{3}{c}{$\begin{array}{c}\text { Días } \\
\text { abiertos }\end{array}$} & \multicolumn{4}{c}{$\begin{array}{c}\text { Edad primer parto } \\
\text { (meses) }\end{array}$} & \multicolumn{2}{c}{$\begin{array}{c}\text { Vida productiva } \\
\text { (meses) }\end{array}$} & \multicolumn{2}{c}{$\begin{array}{c}\text { Producción de leche } \\
\text { (kg leche/día) }\end{array}$} \\
\hline & $\mathbf{X}$ & $\mathbf{n}$ & $\mathbf{E . E}$ & $\mathbf{X}$ & $\mathbf{n}$ & $\mathbf{E . E}$ & $\mathbf{X}$ & $\mathbf{n}$ & $\mathbf{E . E}$ & $\mathbf{X}$ & $\mathbf{n}$ & $\mathbf{E . E}$ \\
\hline J8 & 131,9 & 14274 & 0,78 & 28,4 & 4992 & 0,07 & 37,0 & 2982 & 0,52 & 15,1 & 217357 & 0,01 \\
H2J6 & 124,9 & 5836 & 1,15 & 28,1 & 2195 & 0,10 & 34,5 & 1115 & 0,84 & 16,2 & 81384 & 0,02 \\
H3J5 & 132,1 & 495 & 4,60 & 28,4 & 216 & 0,33 & 30,5 & 99 & 2,39 & 16,1 & 8110 & 0,07 \\
H4J4 & 130,4 & 9124 & 0,98 & 28,8 & 3153 & 0,09 & 38,4 & 1826 & 0,70 & 16,8 & 124793 & 0,02 \\
H5J3 & 126,6 & 396 & 4,46 & 27,9 & 185 & 0,31 & 29,9 & 68 & 3,16 & 17,5 & 5196 & 0,09 \\
H6J2 & 139,0 & 3249 & 1,64 & 29,2 & 1408 & 0,13 & 31,1 & 687 & 1,01 & 17,1 & 48214 & 0,03 \\
H8 & 151,5 & 17290 & 0,76 & 30,2 & 6546 & 0,07 & 36,5 & 4525 & 0,43 & 18,6 & 272487 & 0,01 \\
H6PS2 & 151,4 & 1588 & 2,36 & 29,9 & 556 & 0,28 & 40,2 & 445 & 1,42 & 17,3 & 23135 & 0,04 \\
H5PS3 & 155,3 & 167 & 6,62 & 26,7 & 58 & 0,29 & 44,0 & 48 & 3,55 & 17,6 & 2488 & 0,13 \\
H4PS4 & 142,8 & 2285 & 1,98 & 29,9 & 771 & 0,21 & 43,5 & 582 & 1,18 & 16,5 & 26055 & 0,04 \\
H3PS5 & 164,8 & 390 & 4,51 & 26,8 & 181 & 0,13 & 30,5 & 128 & 2,15 & 17,7 & 6628 & 0,07 \\
H2PS6 & 153,4 & 1014 & 2,70 & 27,4 & 409 & 0,16 & 35,8 & 325 & 1,42 & 16,8 & 14563 & 0,05 \\
PS8 & 148,7 & 2825 & 1,70 & 28,8 & 1060 & 0,11 & 38,4 & 894 & 0,91 & 15,9 & 37767 & 0,03 \\
\hline
\end{tabular}

E.E = Error estándar.

93,3\% para vida productiva y $98,1 \%$ para $\mathrm{kg}$ de leche. En algunas medias de mínimos cuadrados se observaron intervalos de confianza muy amplios, principalmente en los grupos H3J5, H5J3, H5PS3 y H3PS5, ya que en estos grupos es donde hay menor cantidad de información disponible; siendo también donde se observó una mayor variabilidad en rendimiento. A pesar de ésto, el modelo genético fue razonablemente eficiente para explicar las diferencias de rendimiento observadas a nivel de campo entre los grupos raciales analizados.

Para el rasgo días abiertos, en los cruces $\mathrm{H} \times \mathrm{J}$ se observó un efecto aditivo directo muy marcado, ya que los días abiertos tendieron a reducirse conforme se incrementó la fracción de la raza Jersey (Figura 1). Asimismo, se observó un efecto favorable de heterosis directa, cuya magnitud fue de $-6,4 \%$ (Cuadro 5). Estos resultados fueron confirmados por el modelo genético (Cuadro 4) ya que ambos coeficientes, AD_J y HD_J, fueron negativos y significativos, aunque el efecto de heterosis fue solo la mitad del efecto aditivo. Los demás efectos no fueron estadísticamente significativos para este rasgo en los cruces $\mathrm{H} \times \mathrm{J}$.
En los cruces H×PS se observó también un efecto aditivo directo significativo (Cuadro 4) ya que los días abiertos tendieron a bajar conforme aumentó la fracción de la raza Pardo Suizo (Figura 1), aunque de manera menos marcada que en el cruce $\mathrm{H} \times \mathrm{J}$. El estimado de heterosis directa no fue significativo (Cuadro 4), y su magnitud, aunque favorable, fue de tan sólo $-2,23 \%$ (Cuadro 5). Todos los demás efectos no fueron significativos para este rasgo en los cruces $\mathrm{H} \times \mathrm{PS}$.

Varios autores han comparado el rendimiento de híbridos de razas lecheras contra las razas puras paternas. Se ha reportado un estimado favorable de $7,3 \%$ de heterosis directa para días abiertos en cruces $\mathrm{H} \times \mathrm{PS}$ (Cassell y McAllister 2007). Otro estudio ha reportado un estimado de $6,7 \%$ también para cruces H×PS (Dechow et al. 2007). Ambos resultados son similares al obtenido en nuestro estudio para cruces $\mathrm{H} \times \mathrm{J}$, pero mayores al obtenido con $\mathrm{H} \times \mathrm{PS}$. La raza Jersey se ha caracterizado generalmente por su mayor rusticidad y eficiencia reproductiva, en contraposición a las razas de mayor envergadura, por lo que parece razonable que los cruces de $\mathrm{H} \times \mathrm{J}$ tiendan a exhibir mayor nivel de heterosis para días abiertos que los cruces 

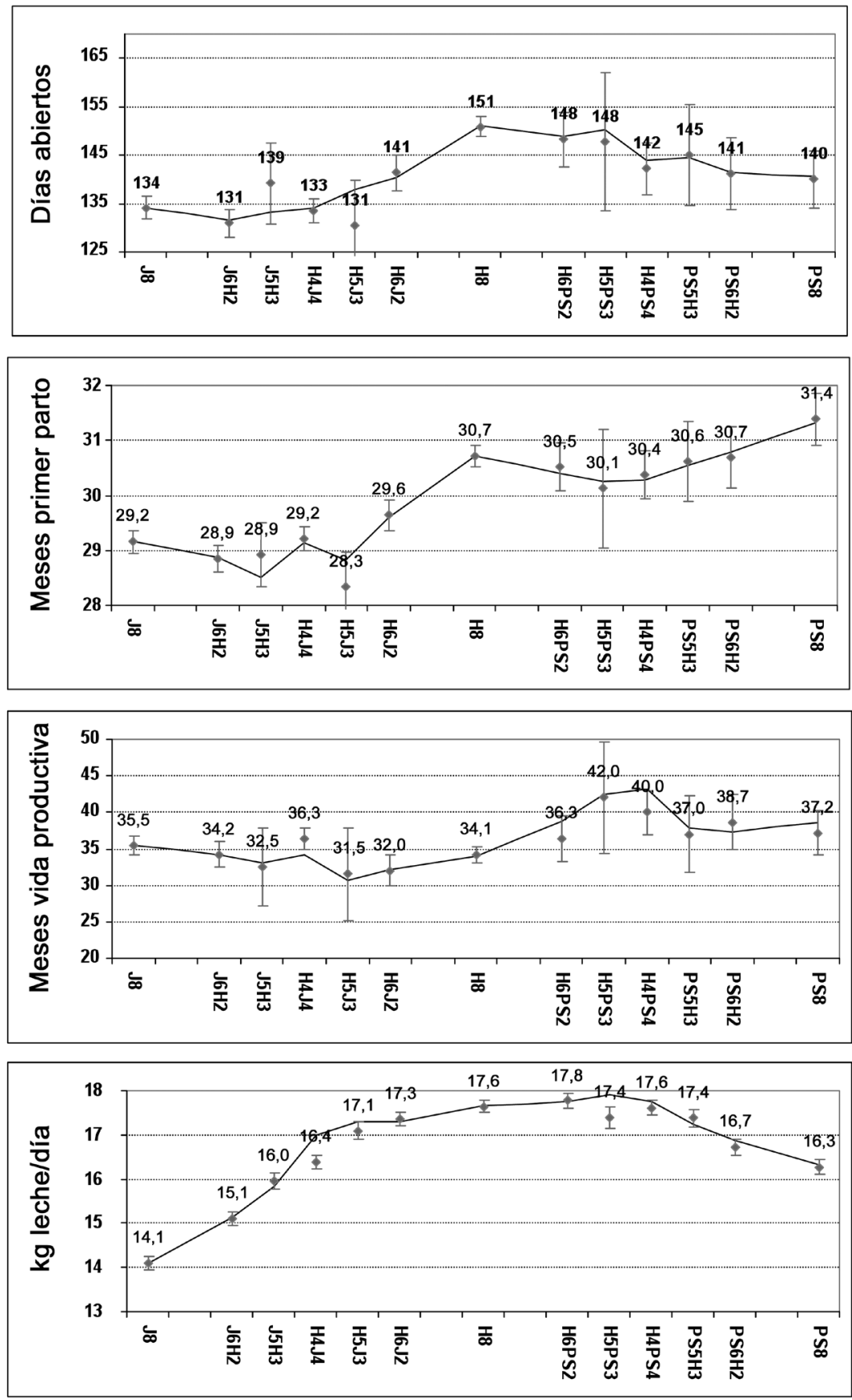

Figura 1. Medias de mínimos cuadrados (e intervalos de confianza 95\%) de rasgos productivos y funcionales obtenidas con un modelo de clasificación racial vs. rendimientos predichos por un modelo genético (línea continua) para razas puras (Holstein,H8; Jersey,J8, y Pardo Suizo, PS8), cruces F1 (H4J4 y H4PS4) y cruces (H2J6, H6J2, H2PS6, H6PS2, H3J5, H5J3, H3PS5, H5PS3). Costa Rica, periodo 1990-2009. 
H×PS. Un estudio reportó un estimado desfavorable de $9,4 \%$ de heterosis en días abiertos para cruces Holstein $\times$ Guernsey (Touchberry 1992). Esto ratifica que los niveles de heterosis pueden variar considerablemente dependiendo de las razas consideradas y el ambiente en que se desenvuelvan.

Para el rasgo edad a primer parto en los cruces $\mathrm{H} \times \mathrm{J}$ se observa nuevamente un efecto aditivo directo marcado ya que la edad tendió a disminuir conforme aumentó la fracción de raza Jersey (Figura 1). Este efecto se confirmó en el modelo genético ya que el coeficiente $\mathrm{AD} \_\mathrm{J}$ es negativo y significativo (Cuadro 4). El efecto de heterosis directa también fue favorable y significativo (Cuadro 4), aunque el coeficiente respectivo fue menos de la mitad del coeficiente aditivo. El porcentaje de heterosis directa obtenido fue de tan solo $-2,5 \%$ (Cuadro 5). No se observaron efectos maternos aditivos significativos, aunque sí se observó heterosis materna significativa y desfavorable. Por el contrario, se observó un inesperado efecto de recombinación favorable y significativo (Cuadro 4).

En el caso de los cruces $\mathrm{H} \times \mathrm{PS}$ el efecto aditivo directo sobre edad al parto no fue tan marcado, pero sí fue significativo (Cuadro 4), lo que indica una ligera tendencia de aumento en edad al parto a mayor fracción de raza PS (Figura 1). El efecto de heterosis directa también fue significativo, lo que indica una menor edad al parto para las F1 (Cuadro 4). El porcentaje de heterosis directa fue de $-2,2 \%$ (Cuadro 5). Los demás efectos no fueron estadísticamente significativos.

Se ha reportado heterosis directa favorable de 3,5\% para edad al parto en cruces $\mathrm{H} \times \mathrm{PS}$ (Cassell y McAllister 2007), ligeramente superior al encontrado en este estudio. Otra investigación encontró un estimado favorable de $3,45 \%$ de heterosis directa para el mismo cruce, y un desfavorable de 2,34\% de recombinación (Dechow et al. 2007). En cruces Holstein×Guernsey se han reportado niveles de heterosis desfavorables de $1,1 \%$ en edad al parto (Touchberry 1992). La raza Jersey, al ser una raza de menor envergadura, ha sido reconocida por su mayor precocidad, por lo que es razonable esperar un mayor nivel de heterosis en los cruces $\mathrm{H} \times \mathrm{J}$, en comparación con cruces entre razas de mayor envergadura.

En cuanto al efecto favorable inesperado de recombinación observado en $\mathrm{H} \times \mathrm{J}$ puede ser debido a la menor información disponible para algunos grupos raciales; así como a la alta variabilidad en rendimiento

Cuadro 4. Coeficientes de regresión para efectos aditivos directos (AD_J, AD_PS) y maternos (AM_J,AM_PS), heterosis directa (HD_J, HD_PS) y materna (HM_J, HM_PS) y efectos de recombinación (R_J, R_PS), estimados a partir de un modelo genético. Costa Rica, periodo 1990-2009.

\begin{tabular}{lcccccccccccc}
\hline Efecto & \multicolumn{3}{c}{ Días abiertos } & \multicolumn{4}{c}{ Edad primer parto } & \multicolumn{3}{c}{ Vida productiva } & \multicolumn{3}{c}{ Kilogramos de leche } \\
\cline { 2 - 13 } & $\boldsymbol{\beta}^{1}$ & $\mathbf{E . E}^{2}$ & $\mathbf{P}^{\mathbf{3}}$ & $\boldsymbol{\beta}$ & $\mathbf{E . E}$ & $\mathbf{P}$ & $\boldsymbol{\beta}$ & $\mathbf{E . E}$ & $\mathbf{P}$ & $\boldsymbol{\beta}$ & $\mathbf{E . E}$ & $\mathbf{P}$ \\
\hline AD_J & $-17,51$ & 2,35 & $<0,01$ & $-1,48$ & 0,18 & $<0,01$ & 4,22 & 1,48 & $<0,01$ & $-4,30$ & 0,03 & $<0,01$ \\
AM_J & 0,65 & 2,12 & 0,76 & $-0,09$ & 0,16 & 0,58 & $-2,79$ & 1,35 & 0,04 & 0,74 & 0,03 & $<0,01$ \\
HD_J & $-8,87$ & 1,31 & $<0,01$ & $-0,76$ & 0,10 & $<0,01$ & 0,79 & 0,82 & 0,34 & 0,72 & 0,02 & $<0,01$ \\
HM_J & $-5,08$ & 12,81 & 0,69 & 1,87 & 0,88 & 0,04 & 8,05 & 8,61 & 0,35 & $-0,67$ & 0,16 & $<0,01$ \\
R_J & 11,87 & 50,87 & 0,82 & $-8,76$ & 3,51 & 0,01 & $-40,20$ & 34,20 & 0,24 & 2,54 & 0,65 & $<0,01$ \\
& & & & & & & & & & & & \\
AD_P & $-14,62$ & 4,18 & $<0,01$ & 0,78 & 0,33 & 0,02 & $-2,99$ & 2,32 & 0,20 & $-1,77$ & 0,06 & $<0,01$ \\
AM_PS & 4,30 & 3,82 & 0,26 & $-0,18$ & 0,29 & 0,53 & 7,57 & 2,17 & $<0,01$ & 0,42 & 0,05 & $<0,01$ \\
H_PS & $-3,95$ & 2,37 & 0,09 & $-0,65$ & 0,19 & $<0,01$ & 3,03 & 1,39 & 0,03 & 0,56 & 0,04 & $<0,01$ \\
HM_PS & $-14,60$ & 17,25 & 0,40 & 0,23 & 1,19 & 0,84 & $-5,47$ & 8,83 & 0,54 & $-0,53$ & 0,22 & 0,01 \\
R_PS & 63,62 & 68,49 & 0,35 & $-1,39$ & 4,72 & 0,77 & 22,92 & 35,27 & 0,52 & 2,28 & 0,86 & $<0,01$ \\
\hline
\end{tabular}

${ }^{1} \beta$ Coeficiente de regresión.

${ }^{2}$ E.E $=$ Error estándar.

${ }^{3} \mathrm{P}$ es la probabilidad de que $\mathrm{H}_{0}: \beta=0$ sea verdadera. 
Cuadro 5. Medias de Mínimos Cuadrados (MMC) para grupos F1 y porcentajes de heterosis para rasgos productivos y funcionales en cruces Holstein×Jersey y Holstein×Pardo Suizo. Costa Rica, periodo 1990-2009.

\begin{tabular}{lcccc}
\hline Rasgo & $\begin{array}{c}\text { MMC } \\
\text { Grupo H4J4 }\end{array}$ & $\begin{array}{c}\text { Heterosis } \\
(\boldsymbol{\%})\end{array}$ & MMC & Heterosis \\
& Grupo H4PS4 & 145,4 & $-2,23$ \\
\hline Días abiertos & 142,5 & $-6,36$ & 31,1 & $-2,17$ \\
Meses edad primer parto & 29,9 & $-2,46$ & 35,7 & 12,2 \\
Meses vida productiva & 34,8 & 4,40 & 17,0 & 3,8 \\
Producción de leche (kg/día) & 15,9 & 3,20 & & \\
\hline
\end{tabular}

${ }^{1}$ MMC: Media de Mínimos Cuadrados.

que se observa en generaciones posteriores a la F1. Esto se aprecia en las mayores desviaciones observadas entre los valores predichos por el modelo en estos grupos con respecto a los obtenidos utilizando el modelo de clasificación racial (Figura 1).

En cuanto al rasgo vida productiva los resultados también fueron distintos en ambos tipos de cruces. Para éstos $\mathrm{H} \times \mathrm{J}$ se observó una tendencia irregular (Figura 1), con mayor rendimiento de la F1 en comparación con las razas puras, pero rendimientos inferiores para los retrocruces. Aunque el estimado de heterosis directa fue 4,4\% (Cuadro 5), no fue significativo de acuerdo con el modelo genético. Sólo los efectos aditivos (directo y materno) fueron significativos (Cuadro 4).

En el caso de los cruces $\mathrm{H} \times \mathrm{PS}$ el comportamiento es también irregular (Figura 1), observándose una mayor vida productiva de la F1 en comparación con las razas puras, con rendimientos irregulares en los retrocruces posteriores. La amplitud de los intervalos de confianza, sobre todo en los grupos 5/8, dificultó la observación de tendencias. La variable vida productiva está ampliamente influenciada por diversos factores ambientales y de manejo. Aún así, se observó un efecto aditivo materno significativo que se demuestra en una tendencia al aumento en vida productiva a mayor fracción de raza Pardo Suizo en las madres (Cuadro 4).

El efecto de heterosis directa fue significativo (Cuadro 4) pero el coeficiente estimado por el modelo genético fue mucho menor al aditivo materno. Esto significa que el alto porcentaje de heterosis $(12,2 \%)$ observado a partir del modelo de clasificación racial, podría estar en parte confundido por el efecto aditivo materno.

Para comprobar esta hipótesis se calcularon los promedios de vida productiva de los cruces F1 recíprocos, siendo 47,9 $\mathrm{m}$ para las vacas $\mathrm{F} 1$ hijas de madres Pardo Suizo y 31,8 m para las hijas de madres Holstein. Esto significa que la mayor vida productiva observada en el grupo F1 puede ser debida en realidad a un efecto materno aditivo de la raza Pardo Suizo y no a un efecto de heterosis directa, como lo sugiere el modelo de clasificación racial. Los demás efectos no fueron significativos en los cruces $\mathrm{H} \times \mathrm{PS}$.

Estimados de heterosis para vida productiva son escasos en la literatura. Un estudio reportó un promedio de 1,2\% de heterosis para vida productiva en cruces entre distintas razas lecheras (Van Raden y Sanders 2003). Otro estudio encontró una vida productiva significativamente mayor para cruces $\mathrm{H} \times \mathrm{PS}$ (3,2 años) en comparación con estimados de 2,8 para Pardo Suizo, Jersey y cruces $\mathrm{H} \times \mathrm{J}, 2,7$ para Holstein y 2,5 para Guernsey (Cedeño y Vargas 2004).

En cuanto al rasgo $\mathrm{kg}$ de leche, en los cruces $\mathrm{H} \times \mathrm{J}$ se observó un efecto aditivo directo marcado (Figura 1) ya que la producción tendió a disminuir conforme aumentó la fracción de raza Jersey. Este efecto fue significativo de acuerdo con el modelo genético (Cuadro 4). En este caso se observa también un efecto aditivo materno favorable a la raza Jersey. Al calcular las medias para los cruces $\mathrm{F} 1$ recíprocos se obtuvo $17,5 \mathrm{~kg}$ para las F1 hijas de madres Jersey y $16,9 \mathrm{~kg}$ para las F1 hijas de madres Holstein.

Otro estudio también reportó mayores producciones cuando la raza materna fue Jersey (Van Raden y Sanders 2003). Sin embargo, esta diferencia también podría atribuirse a una mayor selección dentro de los toros Holstein en comparación con los Jersey, efecto que no fue posible cuantificar en este estudio.

El efecto de heterosis directa fue favorable y significativo para $\mathrm{kg}$ de leche (Cuadro 4), aunque la 
magnitud del coeficiente respectivo fue solo una sexta parte del aditivo. Se obtuvo un $3,2 \%$ de heterosis de acuerdo con el modelo de clasificación (Cuadro 5). El efecto de heterosis materna fue significativo pero desfavorable, sin embargo es contrarrestado por un mayor efecto de recombinación que, contrario a lo esperado, fue positivo en vez de negativo (Cuadro 4). Esto puede apreciarse en la Figura 1 ya que los rendimientos de los retrocruces tendieron a desviarse positivamente con respecto a la F1.

En los cruces H×PS también se observó un efecto aditivo marcado, con una tendencia a la reducción de producción conforme aumentó la fracción de raza Pardo Suizo (Figura 1). El efecto de heterosis directa fue favorable y significativo (Cuadro 4), pero la magnitud del coeficiente fue solo una tercera parte del aditivo. El porcentaje de heterosis obtenido del modelo de clasificación fue de 3,8\% (Cuadro 5). Los efectos de heterosis materna y recombinación también fueron significativos y mostraron el mismo patrón observado en cruces $\mathrm{H} \times \mathrm{J}$.

En cruces entre razas $\mathrm{H} \times \mathrm{J}$ en Nueva Zelanda, se reportaron niveles de heterosis directa entre 4 y $4,5 \%$ para producción de leche, grasa y proteína (LópezVillalobos et al. 2000a), similares a los encontrados en nuestro estudio para $\mathrm{kg}$ de leche. En otro estudio se reportaron niveles de heterosis de 3,4\% para leche y $4,4 \%$ para grasa en cruces $\mathrm{H} \times \mathrm{J}$ (Van Raden y Sanders 2003). Otros autores han encontrado niveles más elevados de heterosis en cruces entre razas lecheras, por arriba de 6\%, para los mismos rasgos (Touchberry
1992, Dechow et al. 2007, Cassell y McAllister 2007).

Los estimados favorables de recombinación obtenidos contradicen el efecto negativo que generalmente se atribuye a la epistasis. Sin embargo, otra investigación también reportó efectos favorables de recombinación para variables productivas (Van Raden y Sanders 2003), los cuales atribuyeron en parte a la inadecuada estructura de los datos y al uso de un modelo genético incompleto. Es posible que para una estimación más precisa del efecto de recombinación, sea necesario contar con padres y madres de ambas razas en los retrocruces, ya que de lo contrario puede haber confusión parcial entre efectos de recombinación y heterosis materna (Madalena (2001). En este estudio todos los retrocruces incluidos fueron toro puroxvaca $(\mathrm{F} 1 \mathrm{o} 3 / 4)$, lo que pudo contribuir a los estimados positivos de recombinación. Otra posible explicación podría ser algún efecto ambiental no identificado ligado al uso de las madres cruzadas, principalmente amamantamiento prolongado en algunos de estos sistemas.

Al combinar los rasgos anteriores en un estimado de producción de leche por día de vida se obtuvo un estimado de heterosis directa de 7,9\% para $\mathrm{H} \times \mathrm{J}$. Aún así, la raza Holstein tuvo un rendimiento superior a los cruces $\mathrm{H} \times \mathrm{J}$ (Figura 2), lo que se debe a que su mayor producción compensa su mayor edad al parto y días abiertos. Sin embargo, la diferencia con la F1 fue mínima (7,11 vs. 7,03). Se observa también en la Figura 2 la marcada reducción de rendimiento en los retrocruces en comparación con la F1, lo que confirma

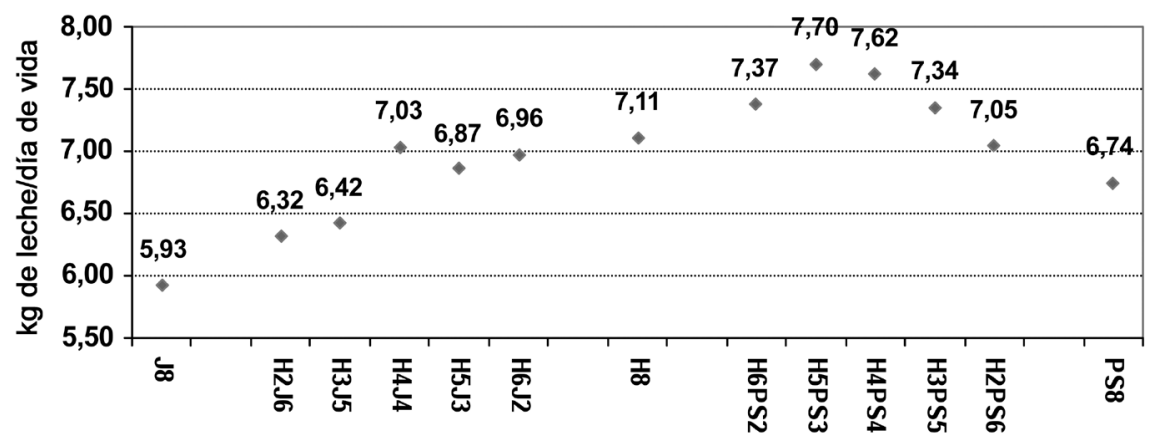

Figura 2. Producción de leche $(\mathrm{kg})$ por día de vida para razas puras (Holstein, H8; Jersey, J8; y Pardo Suizo, PS8), cruces F1 (H4J4 y H4PS4) y cruces (H2J6, H6J2, H2PS6, H6PS2, H3J5, H5J3, H3PS5, H5PS3). Costa Rica, periodo 1990-2009. 
la pérdida de heterosis que generalmente se observa en generaciones posteriores a la F1.

En cuanto a los cruces $\mathrm{H} \times \mathrm{PS}$, la impresión general es que este cruce tiene un efecto más positivo que el observado en $\mathrm{H} \times \mathrm{J}$ (Figura 2). El estimado de heterosis directa fue en este caso de $10,1 \%$. Se observó un mayor rendimiento de todos los cruces, excepto H2PS6, sobre las razas puras. Esta ventaja del $\mathrm{H} \times \mathrm{PS}$ en comparación con los $\mathrm{H} \times \mathrm{J}$, en términos del presente estudio, radica en que presenta un mejor equilibrio entre rendimiento productivo y reproductivo; lo que se traduce en mayor producción por día de vida.

Según se observa en la Figura 2, el uso de cruzamientos $\mathrm{H} \times \mathrm{J}$ podría parecer una alternativa atractiva para un hato Jersey, no así para un hato Holstein. De manera interesante, a nivel de campo es más común encontrar hatos Holstein que introducen cruces con Jersey con el fin de incrementar la producción de grasa y sólidos, lo cual no se justificaría plenamente de acuerdo con el presente estudio. Por otro lado, de acuerdo con los resultados, el uso de cruzamientos H×PS sí podría considerarse como una opción atractiva tanto para hatos Holstein como Pardo Suizo.

Esta comparación, sin embargo, dista de ser exhaustiva, ya que no incluye otros rasgos de importancia económica, como la producción de grasa y proteína en leche, la eficiencia de conversión alimenticia, crecimiento, valores de desecho, etc. con información disponible sobre producción de grasa, en $\mathrm{kg}$ por día de vida. Se observó una pequeña ventaja del F1 $\mathrm{H} \times \mathrm{J}$ contra el Holstein puro $(0,31 \mathrm{~kg}$ vs. $0,29 \mathrm{~kg})$, pero las producciones de los retrocruces siguen siendo iguales o menores que el Holstein puro. No se contó con información de grasa en los cruces $\mathrm{H} \times \mathrm{PS}$.

Existen pocos estudios que comparen rendimientos de por vida entre cruces lecheros versus razas puras. En cruces de Holstein $\times$ Ayrshire, se ha reportado niveles de heterosis de $16,6 \%$ para producción de leche de por vida y $20,6 \%$ para ganancia neta anual descontada, siendo los rendimientos de la F1 mayores que para ambas razas puras (McAllister et al. 1994). Estos resultados son muy superiores a lo que sugiere el presente trabajo. Se han utilizado modelos de simulación para comparar el ingreso neto/vaca/año e ingreso neto/hectárea/año basados en los niveles reales de heterosis observados en cruces $\mathrm{H} \times \mathrm{J}$ bajo condiciones de producción extensiva en Nueva Zelanda, asumiendo un sistema de pago con penalización para fluído (López-Villalobos et al. 2000b). El resultado de la simulación pronosticó un mayor ingreso neto en hatos con cruces rotacionales que en hatos puros. En el presente estudio no se realizaron comparaciones a nivel económico, pero los parámetros obtenidos podrían servir de base para este tipo de estimaciones. Sin embargo, se debe tener presente que estas comparaciones pueden variar sustancialmente dependiendo de las circunstancias preponderantes, tales como zona de vida, tipo de alimentación, sistemas de crianza o sistemas de pago de leche. Además, debe considerarse que un hato bajo un sistema de cruce rotacional en etapa de transición está formado por animales con proporciones raciales muy variables, las cuales deben ser consideradas a la hora de realizar dichas comparaciones.

\section{LITERATURA CITADA}

Ahlborn-Breier, G; Hohenboken, WD. 1991. Additive and nonadditive genetic effects on milk production in dairy cattle: evidence for major individual heterosis. Journal of Dairy Science 74:592-602.

Cassell, B; McAllister, J. 2007. Dairy crossbreeding: Why and how. Dairy Guidelines no. 404-093 Virginia Cooperative Extension. 5 p.

Cedeño, DA; Vargas, B. 2004. Efecto de la raza y el manejo sobre la vida productiva del bovino lechero en Costa Rica. Archivos de Zootecnia 53:129-140.

Dechow, CD; Rogers, GW; Cooper, JB; Phelps, MI; Mosholder, AL. 2007. Milk, fat, protein, somatic cell score, and days open among Holstein, Brown Swiss, and their crosses. Journal of Dairy Science 90:3542-3549.

Holdridge, LD. 2000. Ecología basada en zonas de vida. 1ra. ed. 5ta reimpresión. Traducido por Humberto Jiménez Saa. IICA. San José, Costa Rica. 216 p.

López Villalobos, N; Garrick, DJ; Blair, HT; Holmes, CW. 2000a. Possible effects of 25 years of selection and crossbreeding on the genetic merit and productivity of New Zealand dairy cattle. Journal of Dairy Science 83:154-163.

López Villalobos, N; Garrick, DJ; Holmes, CW; Blair, HT; Spelman, RJ. 2000b. Profitabilities of some mating systems for dairy herds in New Zealand. Journal of Dairy Science 83:144-153.

Madalena, F. 1993. La utilización sostenible de hembras F1 en la producción del ganado lechero tropical. Estudio FAO producción y sanidad animal. p. 111-112. 
Madalena, F. 2001. Consideraciones sobre modelos para la predicción del desempeño de cruzamientos bovinos. Archivos Latinoamericanos de Producción Animal 9:108-117.

Madalena, F. 2008. How sustainable are the breeding programs of the global main stream dairy breeds? (en línea). The Latin American situation. Livestock Research for Rural Development 20(2). Consultado 1 marzo 10. Disponible en http://www.lrrd.org/lrrd20/2/mada20019.htm

McAllister, AJ; Lee, AJ, Batra, TR; Lin, CY; Roy, GL; Vesely, JA; Wauthy, JM; Winter, KA. 1994. The influence of additive and non-additive gene action on lifetime yields and profitability in dairy cattle. Journal of Dairy Science 77:2400-2414.

McAllister, AJ. 2002. Is crossbreeding the answer to questions of dairy breed utilization? Journal of Dairy Science 85:2352-2357.

Noordhuizen, JPTM; Buurman, J. 1984. Veterinary automated management and production control program for dairy farms (VAMPP). The application of MUMPS for data processing. Veterinary Quarterly 6:62-77.
Pérez, E; Baayen, MT; Capella, E; Barkema, H. 1989. Development of a livestock information system for Costa Rica. In Kuil, H; Palin, RW; Huhn, JE. eds. Livestock Production and Diseases in the Tropics. Proceedings $4^{\text {th }}$ International Conference Inst. Trop. Vet. Med. Utrecht; The Netherlands. p. 221-224.

SAS Inst. Inc. 2009. SAS/STAT® User's guide: Statistics; Version 9.2. Cary; NC, USA.

Swan, AA; Kinghorn, BP. 1992. Evaluation and explotation of crossbreeding in dairy cattle. Journal of Dairy Science 75:624-639.

Touchberry, RW. 1992. Crossbreeding effect in dairy cattle: The Illinois experiment; 1949 to 1969 . Journal of Dairy Science 75:640-667.

Van Raden, P. 1992. Accounting for inbreeding and crossbreeding in genetic evaluation of large populations. Journal of Dairy Science 75:3136-3144.

Van Raden, PM; Sanders, AH. 2003. Economic merit of crossbred and purebred US dairy cattle. Journal of Dairy Science 86:1036-1044. 